\title{
A REMARK ON WEIGHTED BERGMAN KERNELS ON ORBIFOLDS
}

\author{
Xianzhe Dai, Kefeng Liu and Xiaonan Ma
}

\begin{abstract}
In this note, we explain that Ross-Thomas' result [4, Theorem 1.7] on the weighted Bergman kernels on orbifolds can be directly deduced from our previous result [1]. This result plays an important role in the companion paper [5] to prove an orbifold version of Donaldson theorem.
\end{abstract}

In two very interesting papers $[4,5]$, Ross-Thomas describe a notion of ampleness for line bundles on Kähler orbifolds with cyclic quotient singularities which is related to embeddings in weighted projective spaces. They then apply [4, Theorem 1.7] to prove an orbifold version of Donaldson theorem [5]. Namely, the existence of an orbifold Kähler metric with constant scalar curvature implies certain stability condition for the orbifold. In these papers, the result [4, Theorem 1.7] on the asymptotic expansion of Bergman kernels plays a crucial role.

In this note, we explain how to directly derive Ross-Thomas' result [4, Theorem 1.7] from Dai-Liu-Ma [1, (5.25)], provided Ross-Thomas condition [4, (1.8)] on $c_{i}$ holds. Since in [1, Section 5], we state our results for general symplectic orbifolds, in what follows, we will just use the version from [2, Theorem 5.4.11], where MaMarinescu wrote them in detail for Kähler orbifolds. We will use freely the notation in $[2$, Section 5.4]. We assume also the auxiliary vector bundle $E$ therein is $\mathbb{C}$.

Let $(X, J, \omega)$ be a compact $n$-dimensional Kähler orbifold with complex structure $J$, and with singular set $X_{\text {sing. }}$. Let $\left(L, h^{L}\right)$ be a holomorphic Hermitian proper orbifold line bundle on $X$. Let $\nabla^{L}$ be the holomorphic Hermitian connections on $\left(L, h^{L}\right)$ with curvature $R^{L}=\left(\nabla^{L}\right)^{2}$.

We assume that $\left(L, h^{L}, \nabla^{L}\right)$ is a prequantum line bundle, i.e.,

$$
R^{L}=-2 \pi \sqrt{-1} \omega .
$$

Let $g^{T X}=\omega(\cdot, J \cdot)$ be the Riemannian metric on $X$ induced by $\omega$. Let $\nabla^{T X}$ be the Levi-Civita connection on $\left(X, g^{T X}\right)$. We denote by $R^{T X}=\left(\nabla^{T X}\right)^{2}$ the curvature, by $r^{X}$ the scalar curvature of $\nabla^{T X}$. For $x \in X$, set $d\left(x, X_{\text {sing }}\right):=\inf _{y \in X_{\text {sing }}} d(x, y)$ the distance from $x$ to $X_{\text {sing }}$.

For $p \in \mathbb{N}$, the Bergman kernel $P_{p}\left(x, x^{\prime}\right)\left(x, x^{\prime} \in X\right)$ is the smooth kernel of the orthogonal projection from $\mathscr{C}^{\infty}\left(X, L^{p}\right)$ onto $H^{0}\left(X, L^{p}\right)$, with respect to the Riemannian volume form $d v_{X}\left(x^{\prime}\right)$.

Theorem 0.1 ([1, Theorem 1.4], [2, Theorem 5.4.10]). There exist smooth coefficients $\boldsymbol{b}_{r}(x) \in \mathscr{C}^{\infty}(X)$ which are polynomials in $R^{T X}$ and its derivatives with order $\leqslant 2 r-2$ 
at $x$, and $C_{0}>0$ such that for any $k, l \in \mathbb{N}$, there exist $C_{k, l}>0, M \in \mathbb{N}$ with

$$
\begin{aligned}
& \left|\frac{1}{p^{n}} P_{p}(x, x)-\sum_{r=0}^{k} \boldsymbol{b}_{r}(x) p^{-r}\right|_{\mathscr{C} l} \\
& \leqslant C_{k, l}\left(p^{-k-1}+p^{l / 2}\left(1+\sqrt{p} d\left(x, X_{\text {sing }}\right)\right)^{M} e^{-\sqrt{C_{0} p} d\left(x, X_{\text {sing }}\right)}\right),
\end{aligned}
$$

for any $x \in X, p \in \mathbb{N}^{*}$. Moreover,

$$
\boldsymbol{b}_{0}=1, \quad \boldsymbol{b}_{1}=\frac{1}{8 \pi} r^{X} .
$$

In local coordinates, there is a more precise form $[1,(5.25)]$, see also $[2$, Theorem 5.4.11]. Let $\left\{x_{i}\right\}_{i=1}^{I} \subset X_{\text {sing }}$. For each point $x_{i}$ we consider corresponding local charts $\left(G_{x_{i}}, \widetilde{U}_{x_{i}}\right) \rightarrow U_{x_{i}}$ with $\widetilde{U}_{x_{i}} \subset \mathbb{C}^{n}$, such that $0 \in \widetilde{U}_{x_{i}}$ is the inverse image of $x_{i} \in U_{x_{i}}$, and 0 is a fixed point of the finite stabilizer group $G_{x_{i}}$ at $x_{i}$, which acts $\mathbb{C}$-linearly and effectively on $\mathbb{C}^{n}$ (cf. [2, Lemma 5.4.3]). We assume moreover that

$$
B^{\widetilde{U}_{x_{i}}}(0,2 \varepsilon) \subset \widetilde{U}_{x_{i}} \text {, and } X_{\text {sing }} \subset W:=\cup_{i=1}^{I} B^{\widetilde{U}_{x_{i}}}\left(0, \frac{1}{4} \varepsilon\right) / G_{x_{i}} .
$$

Let $\widetilde{U}_{x_{i}}^{g}$ be the fixed point set of $g \in G_{x_{i}}$ in $\widetilde{U}_{x_{i}}$, and let $\widetilde{N}_{x_{i}, g}$ be the normal bundle of $\widetilde{U}_{x_{i}}^{g}$ in $\widetilde{U}_{x_{i}}$. For each $g \in G_{x_{i}}$, the exponential map $\widetilde{N}_{x_{i}, g, \widetilde{x}} \ni Y \rightarrow \exp _{\widetilde{x}} \widetilde{U}_{x_{i}}(Y)$ identifies a neighborhood of $\widetilde{U}_{x_{i}}^{g}$ with $\widetilde{W}_{x_{i}, g}=\left\{Y \in \widetilde{N}_{x_{i}, g},|Y| \leqslant \varepsilon\right\}$. We identify $\left.L\right|_{\widetilde{W}_{x_{i}, g}}$ with $\left.L\right|_{\widetilde{U}_{x_{i}}^{g}}$ by using the parallel transport along the above exponential map. Then the $g$-action on $\left.L\right|_{\widetilde{W}_{x_{i}, g}}$ is the multiplication by $e^{\sqrt{-1} \theta_{g}}$, and $\theta_{g}$ is locally constant on $\widetilde{U}_{x_{i}}^{g}$.

Let $\nabla^{\widetilde{N}_{x_{i}, g}}$ be the connection on $\widetilde{N}_{x_{i}, g}$ induced by the Levi-Civita connection via projection. We trivialize $\widetilde{N}_{x_{i}, g} \simeq \widetilde{U}_{x_{i}}^{g} \times \mathbb{C}^{n_{g}}$ by the parallel transport along the curve $[0,1] \ni t \rightarrow t \widetilde{Z}_{1, g}$ for $\widetilde{Z}_{1, g} \in \widetilde{U}_{x_{i}}^{g}$, which identifies also the metric on $\widetilde{N}_{x_{i}, g}$ with the canonical metric on $\mathbb{C}^{n_{g}}$. If $\widetilde{Z} \in \widetilde{W}_{x_{i}, g}$, we will write $\widetilde{Z}=\left(\widetilde{Z}_{1, g}, \widetilde{Z}_{2, g}\right)$ with $\widetilde{Z}_{1, g} \in \widetilde{U}_{x_{i}}^{g}$, $\widetilde{Z}_{2, g} \in \mathbb{C}^{n_{g}}$. We will denote by $Z$ the corresponding point on the orbifold.

Theorem $0.2\left([1,(5.25)]\right.$, [2, Theorem 5.4.11]). On $\widetilde{U}_{x_{i}}$ as above, there exist polynomials $\mathscr{K}_{r, \widetilde{Z}_{1, g}}\left(\widetilde{Z}_{2, g}\right)$ in $\widetilde{Z}_{2, g}$ of degree $\leqslant 3 r$, of the same parity as $r$, whose coefficients are polynomials in $R^{T X}$ and its derivatives of order $\leqslant r-2$, and a constant $C_{0}>0$ such that for any $k, l \in \mathbb{N}$, there exist $C_{k, l}>0, N \in \mathbb{N}$ such that

$$
\begin{aligned}
& \mid \frac{1}{p^{n}} P_{p}(\widetilde{Z}, \widetilde{Z})-\sum_{r=0}^{k} \boldsymbol{b}_{r}(\widetilde{Z}) p^{-r} \\
& \quad-\left.\sum_{r=0}^{2 k} p^{-\frac{r}{2}} \sum_{1 \neq g \in G_{x_{i}}} e^{\sqrt{-1} \theta_{g} p} \mathscr{K}_{r, \widetilde{Z}_{1, g}}\left(\sqrt{p} \widetilde{Z}_{2, g}\right) e^{-2 \pi p\left\langle\left(1-g^{-1}\right) \widetilde{z}_{2, g}, \overline{\tilde{z}_{2, g}}\right\rangle}\right|_{\mathscr{C}^{l}} \\
& \quad \leqslant C_{k, l}\left(p^{-k-1}+p^{-k+\frac{l-1}{2}}\left(1+\sqrt{p} d\left(Z, X_{\text {sing }}\right)\right)^{N} e^{-\sqrt{C_{0} p} d\left(Z, X_{\text {sing }}\right)}\right),
\end{aligned}
$$

for any $|\widetilde{Z}| \leqslant \varepsilon / 2, p \in \mathbb{N}$, with $\boldsymbol{b}_{r}(\widetilde{Z})$ as in Theorem 0.1 and $\mathscr{K}_{0, \widetilde{Z}_{1, g}}=1$. 
Given a function $f(p, x)$ in $p \in \mathbb{N}$ and $x \in X$, we write $f=\mathcal{O}_{\mathscr{C}^{j}}\left(p^{l}\right)$ if the $\mathscr{C}^{j}$-norm of $f$ is uniformly bounded by $C p^{l}$.

Theorem 0.3. Let $(X, \omega)$ be a compact $n$-dimensional Kähler orbifold with cyclic quotient singularities (i.e., the stabilizer group $G_{x}$ is a cyclic group for any $x \in X$ ), and $L$ be a proper orbifold line bundle on $X$ equipped with a Hermitian metric $h^{L}$ whose curvature form is $-2 \pi \sqrt{-1} \omega$, such that for any $x \in X$, the stabilizer group $G_{x}$ acts on $L_{\widetilde{x}}$ as $\mathbb{Z}_{\left|G_{x}\right|}$-order cyclic group. Fix $N \geq 0$, and $r \geq 0$ and suppose $c_{i}$ are a finite number of positive constants chosen so that if $X$ has an orbifold point of order $m$ then

$$
\frac{1}{m} \sum_{i} i^{k} c_{i}=\sum_{i \equiv u \bmod m} i^{k} c_{i}, \quad \text { for all } u \text { and all } k=0, \ldots, N+r .
$$

Then the function

$$
B_{p}^{\mathrm{orb}}(x):=\sum_{i} c_{i} P_{p+i}(x, x)
$$

admits a global $\mathscr{C}^{2 r}$-expansion of order $N$. That is, there exist smooth functions $b_{0}, \ldots, b_{N}$ on $X$ such that

$$
B_{p}^{\mathrm{orb}}=\sum_{j=0}^{N} b_{j} p^{n-j}+\mathcal{O}_{\mathscr{C}^{2 r}}\left(p^{n-N-1}\right) .
$$

Furthermore, $b_{j}$ are universal polynomials in the constants $c_{i}$ and the derivatives of $\omega$; in particular

$$
b_{0}=\sum_{i} c_{i}, \quad b_{1}=\sum_{i} c_{i}\left(n i+\frac{1}{8 \pi} r^{X}\right) .
$$

Remark 0.1. Theorem 0.3 recovers [4, Theorem 1.7] of Ross-Thomas, where the remainder estimate is $\mathcal{O}_{\mathscr{C}} r\left(p^{n-N-1}\right)$.

We improve here their remainder estimate to $\mathcal{O}_{\mathscr{C}^{2 r}}\left(p^{n-N-1}\right)$ and we get Theorem 0.3 directly from Theorems 0.1 and 0.2 .

Remark 0.2. By Ma-Marinescu [3, (3.30), Remark 3.10], [2, Theorem 4.1.3, Remark 5.4.12], Theorem 0.3 generalizes to any $J$-invariant metric $g^{T X}$ on $T X$. Set $\Theta:=$ $g^{T X}(J \cdot, \cdot)$. The only change is that the coefficients in the expansion become

$$
b_{0}=\frac{\omega^{n}}{\Theta^{n}} \sum_{i} c_{i}, b_{1}=\frac{\omega^{n}}{\Theta^{n}} \sum_{i} c_{i}\left[n i+\frac{r_{\omega}^{X}}{8 \pi}-\frac{1}{4 \pi} \Delta_{\omega} \log \left(\frac{\omega^{n}}{\Theta^{n}}\right)\right],
$$

where $r_{\omega}^{X}, \Delta_{\omega}$ are the scalar curvature and the Bochner Laplacian associated to $g_{\omega}^{T X}=\omega(\cdot, J \cdot)$. Moreover, $(0.7)$ can be taken to be uniform as $\left(h^{L}, g^{T X}\right)$ runs over a compact set.

Proof of Theorem 0.3. Recall that now $G$ is a cyclic group of order $m$. Let $\zeta$ be a generator of $G$. From the local condition for orbi-ample line bundles, $\zeta$ acts on $L_{x_{i}}$ as 
a primitive $m$ th root of unity $\lambda$. Thus in (0.4), $e^{\sqrt{-1} \theta_{\zeta^{u}}}=\lambda^{u}$. For $u \in\{1, \ldots$, $m-1\}$, set

$$
\begin{aligned}
\eta_{u} & =e^{-2 \pi\left\langle\left(1-\zeta^{-u}\right) \widetilde{z}_{2, \zeta^{u}}, \overline{\widetilde{z}}_{2, \zeta^{u}}\right\rangle}, \\
S_{u}(\widetilde{Z}) & =\sum_{i} c_{i} \sum_{j=0}^{2 N+2 r+1}(p+i)^{n-\frac{j}{2}} \mathscr{K}_{j, \widetilde{Z}_{1, \zeta^{u}}}\left(\sqrt{p+i} \widetilde{Z}_{2, \zeta^{u}}\right) \lambda^{u(p+i)} \eta_{u}^{p+i}, \\
\mathcal{S}_{2} & =\sum_{u=1}^{m-1} S_{u}, \quad \mathcal{S}_{1}=\sum_{i} c_{i} \sum_{j=0}^{N+r} \boldsymbol{b}_{j}(\widetilde{Z})(p+i)^{n-j} .
\end{aligned}
$$

Here $Z=z+\bar{z}$, and $z=\sum_{i} z_{i} \frac{\partial}{\partial z_{i}}, \bar{z}=\sum_{i} \bar{z}_{i} \frac{\partial}{\partial \bar{z}_{i}}$ when we consider them as vector fields, and $\left|\frac{\partial}{\partial z_{i}}\right|^{2}=\left|\frac{\partial}{\partial \bar{z}_{i}}\right|^{2}=\frac{1}{2}$. Similarly for $\widetilde{Z}$ (and those with subscripts).

Applying (0.4) for $k=N+r+1$ we obtain for $|\widetilde{Z}| \leq \varepsilon / 2$,

$$
\begin{aligned}
\mid B_{p}^{\mathrm{orb}} & (\widetilde{Z})-\mathcal{S}_{1}-\left.\mathcal{S}_{2}\right|_{\mathscr{C}^{l^{\prime}}} \\
\leqslant & C_{l^{\prime}} p^{n-N-r-2}\left(1+p^{\frac{l^{\prime}+1}{2}}\left(1+\sqrt{p} d\left(Z, X_{\text {sing }}\right)\right)^{M} e^{-\sqrt{C_{0} p} d\left(Z, X_{\text {sing }}\right)}\right) \\
& +\sum_{i} c_{i}(p+i)^{n-N-r-1} \\
& \times\left(\sum_{u=1}^{m-1}\left|\mathscr{K}_{2 N+2 r+2, \widetilde{Z}_{1, \zeta^{u}}}\left(\sqrt{p+i} \widetilde{Z}_{2, \zeta^{u}}\right) \eta_{u}^{p+i}\right|_{\mathscr{C} l^{\prime}}+\left|\boldsymbol{b}_{N+r+1}(\widetilde{Z})\right|_{\mathscr{C} l^{\prime}}\right) .
\end{aligned}
$$

In what follows, we write for simplicity $\widetilde{Z}_{1, \zeta^{u}}$ as $Z_{1, u}$ and $\widetilde{Z}_{2, \zeta^{u}}$ as $Z_{2, u}$. For a function $f(p, Z)$ with $p \in \mathbb{N}$ and $|Z| \leq \varepsilon / 2$ we write $f=\mathcal{O}_{\mathscr{C}^{j}}(g(p, Z))$ if the $\mathscr{C}^{j}$-norm of $f$ in $Z$ can be uniformly controlled by $C|g(p, Z)|$.

Note that $\mathscr{K}_{j, Z_{1, u}}\left(Z_{2, u}\right)$ is a polynomial in $Z_{2, u}$ with the same parity as $j$ and $\operatorname{deg} \mathscr{K}_{j, Z_{1, u}} \leq 3 j$. Denote by $\mathscr{K}_{j, Z_{1, u}, l}$ the $l$-homogeneous part of $\mathscr{K}_{j, Z_{1, u}}$. Then $\mathscr{K}_{j, Z_{1, u}, l}=0$ if $l$ and $j$ are not in the same parity or $l>3 j$. By (0.10),

$$
\begin{aligned}
S_{u}(Z)= & \sum_{i} c_{i} \sum_{j=0}^{2 N+2 r+1} \sum_{l}(p+i)^{n-\frac{j-l}{2}} \mathscr{K}_{j, Z_{1, u}, l}\left(Z_{2, u}\right) \lambda^{u(p+i)} \eta_{u}^{p+i} \\
= & \lambda^{u p} \sum_{j=0}^{2 N+2 r+1}\left\{\left(\sum_{l \geq j-2 n} \sum_{q=0}^{n-\frac{j-l}{2}}+\sum_{l<j-2 n} \sum_{q=0}^{N+r}\right) \mathscr{K}_{j, Z_{1, u}, l}\left(\sqrt{p} Z_{2, u}\right)\right. \\
& \left.\times p^{n-\frac{j}{2}-q}\left(\begin{array}{c}
n-\frac{j-l}{2} \\
q
\end{array}\right) \sum_{i} c_{i} i^{q} \lambda^{u i} \eta_{u}^{p+i}\right\}+\mathcal{O}_{\mathscr{C}^{2 r}}\left(p^{n-N-1}\right) .
\end{aligned}
$$

Here we used $(p+i)^{\gamma}=\sum_{q=0}^{N+r} p^{\gamma-q}\left(\begin{array}{l}\gamma \\ q\end{array}\right) i^{q}+\mathcal{O}\left(p^{\gamma-N-r-1}\right)$ for $\gamma<0$ and the following relations for $r^{\prime}, r^{\prime \prime} \in \mathbb{N}, r^{\prime \prime} \leq l$,

$$
\begin{aligned}
& \mathscr{K}_{j, Z_{1, u}, l}\left(\sqrt{p} Z_{2, u}\right) \eta_{u}^{p}=\mathcal{O}_{\mathscr{C}^{r^{\prime}}}\left(p^{\frac{r^{\prime}}{2}} \eta_{u}^{p / 2}\right), \\
& \mathscr{K}_{j, Z_{1, u}, l}\left(\sqrt{p} Z_{2, u}\right)=\mathcal{O}_{\mathscr{C}^{r^{\prime \prime}}}\left(p^{\frac{l}{2}}\left|Z_{2, u}\right|^{l-r^{\prime \prime}}\right) .
\end{aligned}
$$


In order to prove (0.7) it is sufficient to show that for $0 \leq l \leq N+r, r^{\prime} \leq 2 r$,

$$
w_{l, p}:=\sum_{i} c_{i} i^{l} \lambda^{u i} \eta_{u}^{p+i}=\mathcal{O}_{\mathscr{C}{ }^{\prime}}\left(p^{l-N-r-1+\frac{r^{\prime}}{2}} \eta_{u}^{p / 2}\right) .
$$

In fact, we will prove that $w_{l, p}=\mathcal{O}_{\mathscr{C}^{r^{\prime}}}\left(p^{l-N-r-1+\frac{r^{\prime}}{2}} \eta_{u}^{\left(\frac{3}{4}-\frac{r^{\prime}}{8 r}\right) p}\right)$ for $r^{\prime} \leq 2 r$.

Since $d w_{l, p}=\frac{d \eta_{u}}{\eta_{u}}\left(p w_{l, p}+w_{l+1, p}\right)$, and $\frac{d \eta_{u}}{\eta_{u}}$ has a term $z_{2, u}$ or $\bar{z}_{2, u}$ which can be absorbed by $\eta_{u}^{\frac{1}{8 r} p}$ to get a factor $p^{-1 / 2}$, we see by induction that it is sufficient to prove $w_{l, p}=\mathcal{O}_{\mathscr{C}^{0}}\left(p^{l-N-r-1} \eta_{u}^{\frac{3}{4} p}\right)$. To this end, write

$$
w_{l, p}=\left[\frac{\sum_{i} c_{i} i^{l} \lambda^{u i} \eta_{u}^{i}}{\left(\eta_{u}-1\right)^{N+r-l+1}}\right]\left(\eta_{u}-1\right)^{N+r-l+1} \eta_{u}^{p} .
$$

Since $\lambda$ is a primitive $m$ th root of unity, $\lambda^{u} \neq 1$ if $u \in\{1, \ldots, m-1\}$. From [4, Lemma 3.5], under the condition (0.5), the function $\eta \rightarrow \sum_{i} c_{i} i^{l} \lambda^{u i} \eta^{i}$ has a root of order $N+r-l+1$ at $\eta=1$ and so the term in square brackets is bounded.

For $\left|z_{2, u}\right| \leq \varepsilon$, we have by $(0.10)$,

$$
\left|\eta_{u}-1\right| \leq C\left|z_{2, u}\right|^{2}
$$

By using (0.10) and (0.16) and the fact that $[0, \infty) \ni x \mapsto x^{s} e^{-x}$ is bounded for any $s \geq 0$, we get

$$
\left(\eta_{u}-1\right)^{s} \eta_{u}^{p / 4}=\mathcal{O}\left(p^{-s}\right), \quad \text { for } s \geq 1 .
$$

Thus, $w_{l, p}=\mathcal{O}_{\mathscr{C}^{0}}\left(p^{l-N-r-1} \eta_{u}^{\frac{3}{4} p}\right)$ and $(0.14)$ follows.

Back in (0.12), for $q>N+r$, the corresponding contribution is certainly $p^{n-\frac{j}{2}-q}$. $\mathcal{O}_{\mathscr{C}^{2 r}}\left(p^{r} \eta_{u}^{p / 2}\right)=\mathcal{O}_{\mathscr{C}^{2 r}}\left(p^{n-N-1} \eta_{u}^{p / 2}\right)$, by (0.13). On the other hand, if $0 \leq q \leq N+r$, then, by (0.13) and (0.14), the corresponding contribution is $p^{n-\frac{j}{2}-q} \mathcal{O}_{\mathscr{C}^{2 r}}\left(p^{q-N-r-1+r}\right.$ $\left.\left(1+\sqrt{p}\left|Z_{2, u}\right|\right)^{6 N+6 r+3} \eta_{u}^{p / 2}\right)=\mathcal{O}_{\mathscr{C}^{2 r}}\left(p^{n-N-1} \eta_{u}^{p / 4}\right)$ again. Thus $S_{u}=\mathcal{O}_{\mathscr{C}^{2 r}}\left(p^{n-N-1}\right)$.

From $(0.10)$ and the above argument, $\mathcal{S}_{2}=\mathcal{O}_{\mathscr{C}^{2 r}}\left(p^{n-N-1}\right)$. Combining with (0.10), $(0.11)$ and $(0.13)$, we get $(0.7)$ and $(0.8)$.

\section{References}

[1] X. Dai, K. Liu and X. Ma, On the asymptotic expansion of Bergman Kernel, J. Differential Geom. 72 (2006), 1-41.

[2] X. Ma and G. Marinescu, Holomorphic Morse inequalities and Bergman kernels, progress in mathematics, 254, Birkhäuser Boston Inc., Boston, MA, 2007, 422 pp.

[3] X. Ma and G. Marinescu, Generalized Bergman kernels on symplectic manifolds, Adv. Math. $\mathbf{2 1 7}(4)$ (2008), 1756-1815.

[4] J. Ross and R. Thomas, Weighted Bergman kernels on Orbifolds, J. Differential Geom. 88 (2011), 87-108.

[5] J. Ross and R. Thomas, Weighted projective embeddings, stability of orbifolds, and constant scalar curvature Kähler metrics, J. Differential Geom. 88 (2011), 109-160.

Department of Mathematics, UCSB, CA 93106, USA

E-mail address: dai@math.ucsb.edu 
Center of Mathematical Science, Zhejiang University and Department of Mathematics, UCLA, CA 90095-1555, USA

E-mail address: liu@math.ucla.edu

Université Paris Diderot - Paris 7, UfR de Mathématiques, Case 7012, Site Chevaleret, 75205 PARis Cedex 13, France

E-mail address: ma@math.jussieu.fr 